\title{
A DIRECT AND ACCURATE ADAPTIVE SEMI-LAGRANGIAN SCHEME FOR THE VLASOV-POISSON EQUATION
}

\author{
MARTIN CAMPOS PINTO
}

Université Louis Pasteur, IRMA, CNRS UMR 7501

7, rue René Descartes, 67084 Strasbourg Cedex, France

e-mail: campos@math.u-strasbg.fr

\begin{abstract}
This article aims at giving a simplified presentation of a new adaptive semi-Lagrangian scheme for solving the $(1+1)$ dimensional Vlasov-Poisson system, which was developed in 2005 with Michel Mehrenberger and first described in (Campos Pinto and Mehrenberger, 2007). The main steps of the analysis are also given, which yield the first error estimate for an adaptive scheme in the context of the Vlasov equation. This article focuses on a key feature of our method, which is a new algorithm to transport multiscale meshes along a smooth flow, in a way that can be said optimal in the sense that it satisfies both accuracy and complexity estimates which are likely to lead to optimal convergence rates for the whole numerical scheme. From the regularity analysis of the numerical solution and how it gets transported by the numerical flow, it is shown that the accuracy of our scheme is monitored by a prescribed tolerance parameter $\varepsilon$ which represents the local interpolation error at each time step. As a consequence, the numerical solutions are proved to converge in $L^{\infty}$ towards the exact ones as $\varepsilon$ and $\Delta t$ tend to zero, and in addition to the numerical tests presented in (Campos Pinto and Mehrenberger, 2007), some complexity bounds are established which are likely to prove the optimality of the meshes.
\end{abstract}

Keywords: fully adaptive scheme, semi-Lagrangian method, Vlasov-Poisson system, error estimates, convergence rates, optimal transport of adaptive multiscale meshes

\section{Introduction}

In one space dimension, the Vlasov equation

$$
\begin{gathered}
\partial_{t} f(t, x, v)+v \partial_{x} f(t, x, v)+E(t, x) \partial_{v} f(t, x, v)=0 \\
f(0, \cdot, \cdot)=f_{0} \in \mathcal{C}_{c}^{0}\left(\mathbb{R}^{2}\right), \quad t \geq 0, \quad x, v \in \mathbb{R}
\end{gathered}
$$

coupled with the Poisson equation

$$
\partial_{x} E(t, x)=\int_{\mathbb{R}} f(t, x, v) \mathrm{d} v
$$

describes the evolution of a collisionless electron gas essentially subject to its own electric field, and can be seen as a reduced model for the three-dimensional VlasovMaxell system that describes the dynamic behavior of hot plasmas and particle beams. Here a background ion distribution is assumed to be uniform and constant, and the magnetic effects are neglected in the Lorentz forces. The variables $x$ and $v$ stand for the space position and the velocity, while $f(t, \cdot, \cdot)$ and $E(t, \cdot)$ denote the electron phase space density and the electric field at time $t$, respectively.
In order to save computational resources while approximating the complex and thin structures that may appear in the solutions of the Vlasov equation, several adaptive schemes have been proposed in the past few years, see in particular (Besse et al., 2001; Gutnic et al., 2004; Sonnendrücker et al., 2004), where the authors use moving phase-space grids or interpolatory wavelets. Originated in the semi-Lagrangian method of (Cheng and Knorr, 1976), later revisited by Sonnendrücker et al. (1999), these schemes gave encouraging results in practice, but none was proven to be more efficient than the uniform ones.

Our main objective was therefore to derive from these adaptive strategies a new error estimate that would prove their superiority over any uniform method. By introducing a new adaptive scheme based on multiscale (hierarchical) finite element bases, this objective has been achieved, and we have proven a rigorous error estimate, together with a partial complexity bound, for the adaptive approximation of the problem (1)-(2).

The outline is as follows: in Section 2, the use of adaptive strategies for approximating solutions of nonuniform smoothness-already described by many authors in 
the context of conservation laws, see, e.g., (Cohen et al., 2003; Roussel et al., 2003)—is first justified with basic arguments, and a practical algorithm for designing adaptive $P_{1}$ finite element approximations is given. In Section 3, the principle of the semi-Lagrangian method for solving the Vlasov-Poisson equation is recalled, as well as the error analysis given in (Besse, 2004) in the uniform setting. Section 4 is then devoted to our adaptive scheme. We first address one of the major issues in designing adaptive methods for evolution problems, namely, their ability to predict meshes fine enough to achieve a given accuracy without being much more refined than necessary. We then describe the key feature of our numerical scheme, that is, a new algorithm that transports multiscale meshes along smooth flows in a way that be can be said optimal in the sense of Remark 5. A global error estimate is eventually stated, together with a partial result on the cardinality of the adaptive meshes. For numerical results confirming these theoretical results and exhibiting optimal adaptive meshes obtained in practice, the reader is referred to (Campos Pinto and Mehrenberger, 2007).

\section{Adaptive Finite Elements}

In this section, we recall how adaptive strategies can be proven to be more efficient than uniform ones in terms of convergence rates, and we propose a simple algorithmic setting to perform $P_{1}$ adaptive approximations (for a comprehensive introduction to adaptive approximation, we refer the reader to the excellent review article (DeVore, 1998) see also (Yserentant, 1992) for a detailed presentation of hierarchical finite elements).

2.1. Uniform vs. Adaptive Meshes. We consider here the problem of interpolating some continuous function $g$ known on the unit square $\Omega=[0,1]^{2}$. Since we are to use $P_{1}$ finite elements, i.e., piecewise affine interpolations on conforming triangulations of $\Omega$, we shall think of two different approaches.

The first one consists in using a uniform mesh $\mathcal{K}_{h}$ made of regular shape triangles of diameter $\mathcal{O}(h)$, i.e., triangles $K$ that contain and that are contained in balls of diameters $d_{K}$ and $d_{K}^{\prime}$, respectively, with

$$
C h \leq d_{K} \leq d_{K}^{\prime} \leq C^{\prime} h .
$$

and absolute constants $C$ and $C^{\prime}$. If $g$ belongs to the space $W^{2, \infty}(\Omega)$, i.e., if it is bounded on $\Omega$ and if its secondorder derivatives are also bounded on $\Omega$, the classical estimate

$$
\left\|\left(I-P_{K}\right) g\right\|_{L^{\infty}(K)} \leq C h^{2}|g|_{W^{2, \infty}(K)}
$$

(where $|g|_{W^{2, \infty}(K)}:=\max \left\{\left\|D^{\alpha} g\right\|_{L^{\infty}(K)}:|\alpha|=2\right\}$ denotes the usual Sobolev seminorm), valid on any shape regular triangle $K$ of diameter $\mathcal{O}(h)$, allows us to bound the global interpolation error on $\mathcal{K}_{h}$ by

$$
\left\|\left(I-P_{h}\right) g\right\|_{L^{\infty}(\Omega)} \leq C h^{2}|g|_{W^{2, \infty}(\Omega)} .
$$

Since the cardinality $\#\left(\mathcal{K}_{h}\right)$ of this triangulation is of the order of $h^{-2}$, the above estimate yields

$$
\left\|\left(I-P_{h}\right) g\right\|_{L^{\infty}(\Omega)} \leq C \#\left(\mathcal{K}_{h}\right)^{-1}|g|_{W^{2, \infty}(\Omega)} .
$$

In the perspective of estimating the (a priori) efficiency of a given approximation method, the above inequality, often referred to as a convergence rate, is a key information which somehow expresses the trade-off between the $a c$ curacy of numerical approximations and their complexity, closely related to their computational cost. In particular, the relation (5) makes it possible

- to impose a maximal cardinality on the meshes, while guaranteeing the accuracy of the interpolations or,

- to prescribe a given accuracy on the interpolations, while giving a complexity bound for the associated meshes.

To improve the above trade-off between accuracy and complexity, a different approach consists in designing a mesh which is locally adapted to the function $g$. A way of doing this could be, according to (3), to use bigger triangles (hence larger values of $h$ ) where $g$ has a small $W^{2, \infty}$ seminorm, and smaller ones elsewhere. Intuitively, this should reduce the cardinality of the triangulation, while not increasing much the global interpolation error. A more convenient setting, however, is given by the following local estimate, which is substantially stronger than (3):

$$
\left\|\left(I-P_{K}\right) g\right\|_{L^{\infty}(K)} \leq C|g|_{W^{2,1}(K)},
$$

also valid on any shape regular triangle $K$, and where the scale invariance (i.e., the fact that $C$ does not depend on the diameter $h$ of $K$ ) corresponds to the fact that the Sobolev embedding of $W^{2,1}(K)$ into $L^{\infty}(K)$ is critical (since we have $1 / \infty=1 / 1-2 / d$ in dimension $d=2$ ). According to this estimate, a natural desire is to find a triangulation $\mathcal{K}_{\varepsilon}$ that equilibrates the local seminorms $|g|_{W^{2,1}(K)}$, in the sense that it satisfies

$$
C \varepsilon \leq|g|_{W^{2,1}(K)} \leq C^{\prime} \varepsilon
$$

for any $K \in \mathcal{K}_{\varepsilon}$. Clearly, the associated interpolation $P_{\varepsilon}$ would satisfy

$$
\left\|\left(I-P_{\varepsilon}\right) g\right\|_{L^{\infty}(\Omega)} \leq C \varepsilon
$$

and because summing the left inequalities in (7) yields

$$
\#\left(\mathcal{K}_{\varepsilon}\right) \leq C \varepsilon^{-1}|g|_{W^{2,1}(\Omega)},
$$


the resulting adaptive approximation $\left(\mathcal{K}_{\varepsilon}, P_{\varepsilon} g\right)$ would satisfy

$$
\left\|\left(I-P_{\varepsilon}\right) g\right\|_{L^{\infty}(\Omega)} \leq C \#\left(\mathcal{K}_{\varepsilon}\right)^{-1}|g|_{W^{2,1}(\Omega)} .
$$

Note that this rate is not identical to (5), since it is achieved for functions which are only in $W^{2,1}(\Omega)$. In the case where $g$ is in $W^{2, \infty}$ but has highly nonuniform smoothness, the adaptive approach is also justified by the fact that the quantity $|g|_{W^{2,1}(\Omega)}$ is likely to be much smaller than $|g|_{W^{2, \infty}(\Omega)}$.

2.2. Multiscale Algorithms. From the above arguments, it now appears that an adaptive strategy is likely to yield better results when interpolating some function of nonuniform smoothness. What we did not mention is an algorithm to design a triangulation $\mathcal{K}_{\varepsilon}$ that fulfills the relations (7), and this might in practice be quite a difficult task. For simplicity, we shall therefore restrict ourselves to a particular class of triangulations that are obtained by recursive splittings of dyadic square cells. The resulting multiscale finite elements should then be seen as a compromise between the uniform and the pure adaptive triangulations described above. As it is usual in compromises, we will have to choose between the two inequalities in (7), and because what we are first interested in is the accuracy of the approximations, we will choose the upper one. Nevertheless, we shall see later on that making such a choice still allows us to write down some complexity estimates.

We then begin by introducing multiscale quad meshes, from which we shall later on derive conforming triangulations. To this end, at any level $\ell \in \mathbb{N}$ consider the sets

$$
\mathcal{Q}_{\ell}:=\left\{\alpha_{\ell, i, k} \subset \Omega: 0 \leq i, k \leq 2^{\ell}-1\right\}
$$

made of the dyadic square cells $\alpha_{\ell, i, k}:=\left(i 2^{-\ell},(i+\right.$ 1) $\left.2^{-\ell}\right) \times\left(k 2^{-\ell},(k+1) 2^{-\ell}\right)$ included in $\Omega$, and denote by $\ell(\alpha)$ the level of a given cell $\alpha$. Since the partitions $\mathcal{Q}_{\ell}$ are nested, we may equip them with a natural quadtree structure by defining the children of a given cell $\alpha$ as

$$
\mathcal{C}(\alpha):=\left\{\beta \in \mathcal{Q}_{\ell(\alpha)+1}: \beta \subset \alpha\right\}
$$

and its parent as

$$
\mathcal{P}(\alpha):=\left\{\beta \in \mathcal{Q}_{\ell(\alpha)-1}: \beta \supset \alpha\right\} .
$$

A set $\Lambda \subset \cup_{\ell \geq 0} \mathcal{Q}_{\ell}$ will then be called a tree if it satisfies

$$
\mathcal{Q}_{0}=\{\Omega\} \subset \Lambda \text { and } \alpha \in \Lambda \Longrightarrow \mathcal{P}(\alpha) \in \Lambda,
$$

and if it also satisfies

$$
\alpha \in \Lambda \Longrightarrow \mathcal{C}(\alpha) \subset \Lambda \text { or } \mathcal{C}(\alpha) \cap \Lambda=\emptyset
$$

it will be said consistent with respect to a partitioning principle. In fact, the tree structure should only be seen as a convenient setting for performing algorithmic refinements: just as refining a cell in a mesh consists in replacing it by its children, refining it in a tree $\Lambda$ consists in adding its children to $\Lambda$. The corresponding mesh is then expressed as the leaves of $\Lambda$, i.e., the set

$$
M(\Lambda):=\{\alpha \in \Lambda: \mathcal{C}(\alpha) \cap \Lambda=\emptyset\},
$$

which, according to (9), is a partition of $\Omega$.

Since we are interested in $P_{1}$ interpolations, a natural criterion of refinement is, considering (6), that the local $W^{2,1}$ seminorm of the function $g$ be greater than some prescribed tolerance $\varepsilon$.

Definition 1. ( $\varepsilon$-adapted meshes) A quad mesh $M$ will be said to be $\varepsilon$-adapted to a function $g$ (with respect to the $W^{2,1}$ seminorm) if it satisfies

$$
\sup _{\alpha \in M}|g|_{W^{2,1}(\alpha)} \leq \varepsilon .
$$

This definition can be extended to triangulations.

\section{Algorithm 1. (quad mesh $\varepsilon$-adapted to g)}

- Starting from the root cell $\Lambda_{0}:=\mathcal{Q}_{0}=\{\Omega\}$,

- build for $\ell \geq 0$

$$
\begin{aligned}
\Lambda_{\ell+1}:=\Lambda_{\ell} \cup\{\beta \in \mathcal{C}(\alpha): & \\
& \left.\alpha \in M\left(\Lambda_{\ell}\right), \quad|g|_{W^{2,1}(\alpha)}>\varepsilon\right\}
\end{aligned}
$$

until $\Lambda_{L+1}=\Lambda_{L}$

- and set $\tilde{M}_{\varepsilon}(g):=M\left(\Lambda_{L}\right)$.

Now in order to establish some stability properties for the transport scheme, we will need the quad meshes to be graded, in the sense that any couple $(\alpha, \beta)$ of their cells satisfies

$$
\bar{\alpha} \cap \bar{\beta} \neq \emptyset \Longrightarrow|\ell(\alpha)-\ell(\beta)| \leq 1 .
$$

The smallest graded mesh adapted to $g$ can be seen as the smallest graded refinement of $\tilde{M}_{\varepsilon}(g)$, which is expressed by the following algorithm:

\section{Algorithm 2. (graded mesh $\varepsilon$-adapted to g)}

- Starting from $\Lambda_{0}^{\mathrm{G}}=\Lambda_{L}$, given by Algorithm 1,

- build for $\ell=0, \ldots, L-2$

$$
\begin{aligned}
& \Lambda_{\ell+1}^{\mathrm{G}}:=\Lambda_{\ell}^{\mathrm{G}} \cup\left\{\mathcal{C}(\alpha): \alpha \in \Lambda_{\ell}^{\mathrm{G}}, \ell(\alpha)=\ell\right. \text { and } \\
& \left.\quad \exists \beta \in \Lambda_{\ell}^{\mathrm{G}}, \text { s.t. } \ell(\beta)=\ell(\alpha)+2 \text { and } \bar{\alpha} \cap \bar{\beta} \neq \emptyset\right\},
\end{aligned}
$$

- and set $M_{\varepsilon}(g):=M\left(\Lambda_{L-1}^{G}\right)$. 
Note that we would have $\Lambda_{L}^{\mathrm{G}}=\Lambda_{L-1}^{\mathrm{G}}$ if the former was to be computed. Because $M_{\varepsilon}(g)$ is a refinement of $\tilde{M}_{\varepsilon}(g)$, it is obviously $\varepsilon$-adapted to $g$. But we also have

$$
\#\left(M_{\varepsilon}(g)\right) \leq C \#\left(\tilde{M}_{\varepsilon}(g)\right)
$$

with an absolute constant, see, for instance, Lemma 2.4 in (Dahmen, 1982). As regards the first algorithm, it is easily seen that it converges (i.e., that the level $L$ is finite) as soon as $g$ is in $W^{2,1}(\Omega)$. However, due to the tree structure, it is not possible to write an upper bound for $L$ (hence for $\#\left(\tilde{M}_{\varepsilon}(g)\right)$ ) from the only $W^{2,1}$ seminorm of $g$. This can be seen by thinking of an arbitrary $\phi$ in $W^{2,1}(\Omega)$ and considering the functions $\phi_{j}(x, v):=\phi\left(2^{j} x, 2^{j} v\right)$ which somehow concentrate the $W^{2,1}$ seminorm of $\phi$ in the small domains $\left(0,2^{-j}\right)^{2}$ : as long as $|\phi|_{W^{2,1}(\Omega)}>\varepsilon, j$ levels of refinements are needed in $\tilde{M}_{\varepsilon}\left(\phi_{j}\right)$, while all the seminorms $\left|\phi_{j}\right|_{W^{2,1}(\Omega)}, j \geq 0$, are equal to $|\phi|_{W^{2,1}(\Omega)}$.

Writing down an estimate for the complexity of the $\varepsilon$-adapted meshes is therefore only possible if this hard kind of concentration is prevented. One way of doing so is to demand a bit more smoothness for $g$ : if, e.g., $g$ is in $W^{2, p}(\Omega)$ with $p>1$ (but arbitrarily close to 1 ), then \# $\left(\tilde{M}_{\varepsilon}(g)\right)$ is bounded by $|g|_{W^{2, p}} \varepsilon^{-1}$, up to some multiplicative constant. For a proof, and for another way to prevent this concentration phenomenon, see Chapters 2 and 3 in (Campos Pinto, 2005).

\subsection{Hierarchical $P_{1}$ Finite Elements Based on} Dyadic Meshes. To perform $P_{1}$ interpolation, we shall now associate to any graded quad mesh $M$ a conforming triangulation $\mathcal{K}(M)$ that is equivalent to $M$, in a sense that will be soon made precise. First, a nonconforming triangulation $\tilde{\mathcal{K}}(M)$ is obtained by simply splitting each cell $\alpha$ in $M$ in two triangles, with the following rule: if $\alpha$ is an upper left or a lower right child (of its parent cell), it is splitted into its lower left and upper right halves, and the splitting is symmetric in the other two cases. We can observe in Fig. 1 that unless $M$ is uniform, the resulting triangulation $\tilde{\mathcal{K}}(M)$ is nonconforming. Indeed, when a quad cell $\alpha$ shares an edge with two finer cells $\beta$ and $\lambda$, this gives rise to a big triangle (say $\alpha^{-}$) that shares an edge with two smaller triangles (say $\beta^{-}$and $\lambda^{+}$). Now, because $M$ is graded, it can be seen that this is the only possible configuration where the triangles are nonconforming, and that a conforming triangulation $\mathcal{K}(M)$ can be obtained by simply merging any such pair $\left(\beta^{-}, \lambda^{+}\right)$of small triangles.

Because every cell in $M$ (resp. every triangle in $\mathcal{K}(M)$ ) intersects at most two triangles in $\mathcal{K}(M)$ (resp. two cells in $M$ ), we simultaneously have

$$
\#(\mathcal{K}(M)) \sim \#(M)
$$

( $A \sim B$ meaning that $C A \leq B \leq C^{\prime} A$ holds with absolute constants) and

$$
\sup _{K \in \mathcal{K}(M)}|g|_{W^{2,1}(K)} \sim \sup _{\alpha \in M}|g|_{W^{2,1}(\alpha)}
$$

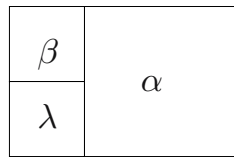

$M$

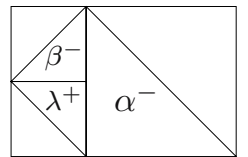

$\tilde{\mathcal{K}}(M)$

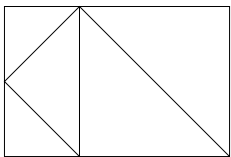

$\mathcal{K}(M)$
Fig. 1. Constructing a conforming triangulation from a graded quad mesh.

for any $g$ in $W^{2,1}$, and this is why we say that $M$ and $\mathcal{K}(M)$ are equivalent. In particular, the piecewise affine interpolation operator $P_{M}$ associated to $M$ via the conforming triangulation $\mathcal{K}(M)$ satisfies

$$
\left\|\left(I-P_{M}\right) g\right\|_{L^{\infty}} \lesssim \sup _{\alpha \in M}|g|_{W^{2,1}(\alpha)}
$$

so that we have

$$
\left\|\left(I-P_{M}\right) g\right\|_{L^{\infty}} \lesssim \varepsilon
$$

as long as $M$ is a graded quad mesh $\varepsilon$-adapted to $g$, which is the case, e.g., for $M_{\varepsilon}(g)$.

\section{Semi-Lagrangian Scheme for the Vlasov Equation}

The principle of the semi-Lagrangian method is to combine Lagrangian transport and interpolation on a given mesh.

3.1. Nonlinear Transport along the Characteristic Curves. Regarding the Vlasov equation (1), the property on which the method is based is the fact that the exact solution $f$ is constant on some particular trajectories, referred to as the characteristic curves of the system. Denoted by $(X, V)(t)=(X, V)(t ; s, x, v)$, these are defined as the unique solution of

$$
(X, V)(s)=(x, v) \quad \text { and } \quad\left\{\begin{array}{l}
X^{\prime}(t)=V(t), \\
V^{\prime}(t)=E(t, X(t)),
\end{array}\right.
$$

so that $(X, V)(t ; s, x, v)$ should be seen as the position at time $t$ of some phase space "particle" that was residing in position $(x, v)$ at time $s$, and whose speed is given by the nonlinear, time depending field $(V, E(t, X))$.

Of course, in order to define these trajectories, we need to know that the solution $f$ does exist and that the electric field $E$ has some smoothness. For what regards the existence of solutions in our case of interest, that is, when the initial data $f_{0}$ are continuous and compactly supported, we refer to a theorem of (Cooper and Klimas, 1980) (based on a previous result of (Iordanskii, 1964)), according to which there exists a continuous solution $f$ that is also compactly supported. 
Remark 1. In order for the solution to remain supported in $\Omega$ during the time interval $[0, T=N \Delta t]$, we can assume in the sequel that $f_{0}$ is supported in a small ball centered in $\Omega$, whose radius depends only on $T$ (see Lemma 2.3 in (Campos Pinto and Mehrenberger, 2007) for details).

Regarding the smoothness of the solutions, estimates for large (but finite) times are also well known, see, e.g., (Glassey, 1996; Raviart, 1985). Basically, what we need to know is that the Sobolev spaces $W^{m, p}$ are preserved by the equation, but precise smoothness estimates are stated and established in (Campos Pinto and Mehrenberger, 2007). Now, from the smoothing effect of the Poisson coupling, as long as the initial condition $f_{0}$ is continuous, the electric field $E$ is a Lipschitz function in space and the characteristic curves are well defined. The Vlasov equation can therefore be written in a Lagrangian form:

$$
\frac{\mathrm{d}}{\mathrm{d} t} f(t, X(t ; 0, x, v), V(t ; 0, x, v))=0, \quad \forall(x, v) \in \Omega .
$$

In particular, a natural way of looking at the solution $f$ in the time interval $[n \Delta t,(n+1) \Delta t]$ is to introduce the backward flow

$$
\mathcal{B}(n):(x, v) \rightarrow(X, V)(n \Delta t ;(n+1) \Delta t, x, v),
$$

so that

$$
f((n+1) \Delta t, \cdot, \cdot)=f(n \Delta t, \cdot, \cdot) \circ \mathcal{B}(n) .
$$

In fact, from the Cauchy-Lipschitz theorem it follows that the flow $\mathcal{B}(n)$ is a $C^{1}$-diffeomorphism from $\mathbb{R}^{2}$ to itself, which will be a key ingredient of our adaptive scheme, as it is in the smoothness analysis of the solutions.

\subsection{Principle of the Semi-Lagrangian Scheme.} Based on this pointwise transport property, the semiLagrangian scheme computes the numerical solution $f_{n+1}$ from $f_{n}$ in three steps (see Fig. 2 below for an illustration with underlying graded quad meshes):

1. approach the exact backward flow $\mathcal{B}(n)$ (12) by some computable $\mathcal{C}^{1}$-diffeomorphism $\mathcal{B}\left[f_{n}\right]$,

2. define an intermediate solution by transporting $f_{n}$ along this flow:

$$
\mathcal{T} f_{n}:=f_{n} \circ \mathcal{B}\left[f_{n}\right]
$$

3. and construct $f_{n+1}$ by interpolating (by piecewise affine elements) this transported solution on some triangulation $\mathcal{K}$.

Remark 2. (computational cost) Like the numerical flow, the intermediate solution $\mathcal{T} f_{n}$ is computable everywhere, but only computed on the nodes of $\mathcal{K}$. The cost of one $f_{n}$

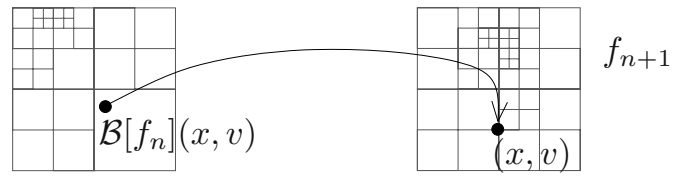

Fig. 2. In the semi-Lagrangian method, the numerical density is transported by (i) following approximated characteristic curves and (ii) interpolating on a given mesh.

iteration is then of the order of $C_{\mathcal{B}} \#(\mathcal{K})$, where $C_{\mathcal{B}}$ is the cost of computing one position $\mathcal{B}\left[f_{n}\right](x, v)$.

Remark 3. (high-order interpolations) Because firstorder interpolations are known to be very diffusive, one may wish to perform higher-order interpolations, which is straightforward as soon as we have a conforming triangulation at hand. Nevertheless, due to the fact that they do not diminish the $L^{\infty}$ norm (as the piecewise affine interpolation does), they lead to a more difficult analysis.

In our particular case, we should mention that the exact flow is approached by a splitting scheme introduced in (Cheng and Knorr, 1976). Let us describe it for completeness: denoting by $E[g]$ the electric field associated to some arbitrary phase space distribution $g$, i.e., satisfying

$$
\partial_{x} E[g](x)=\int g(x, v) \mathrm{d} v,
$$

the splitting consists in defining the one-directional flows

$$
\mathcal{B}_{x}^{1 / 2}(x, v)=(x-v \Delta t / 2, v)
$$

and

$$
\mathcal{B}_{v}[g](x, v)=(x, v-E[g] \Delta t) .
$$

The corresponding (partial) transport operators are then

$$
\mathcal{T}_{x}^{1 / 2}: g \rightarrow g \circ \mathcal{B}_{x}^{1 / 2} \text { and } \mathcal{T}_{v}: g \rightarrow g \circ \mathcal{B}_{v}[g],
$$

and the full transport operator is $\mathcal{T}=\mathcal{T}_{x}^{1 / 2} \mathcal{T}_{v} \mathcal{T}_{x}^{1 / 2}$. Following this principle, we obtain a computable approximation (13), where the backward flow $\mathcal{B}\left[f_{n}\right]:(x, v) \rightarrow$ $(\tilde{x}, \tilde{v})$ is given by

$$
\left\{\begin{array}{l}
\tilde{x}=x-v \Delta t+\left(\Delta t^{2} / 2\right) E\left[\mathcal{T}_{x}^{1 / 2} f_{n}\right](x-v \Delta t / 2), \\
\tilde{v}=v-\Delta t E\left[\mathcal{T}_{x}^{1 / 2} f_{n}\right](x-v \Delta t / 2) .
\end{array}\right.
$$

In (Campos Pinto and Mehrenberger, 2007), it is proved that this splitting scheme is (locally) third-order accurate in time, as the error resulting from the approximation of the exact characteristics - if ever performedwould be bounded by

$$
\|\mathcal{B}(n)(x, v)-\mathcal{B}[f(n \Delta t)](x, v)\|_{\ell^{2}\left(\mathbb{R}^{2}\right)} \leq C \Delta t^{3}
$$

uniformly in $(x, y)$, as long as the initial density $f_{0}$ (and thus $f(n \Delta t))$ is in $W^{1, \infty}\left(\mathbb{R}^{2}\right)$. 
Remark 4. In practice, the numerical electric field is thus given by the one-dimensional integration (14) of a numerical approximation to $\mathcal{T}_{x}^{1 / 2} f_{n}$ (see also Remark 6).

3.3. Error Analysis in the Uniform Setting. In (Besse, 2004), an a-priori error estimate was given for the semi-Lagrangian method in the case where $\mathcal{K}$ is a fixed conforming, shape regular triangulation $\mathcal{K}_{h}$, and where the initial data $f_{0}$ are in $W^{2, \infty}$. The numerical scheme is then

$$
\mathbb{S}_{h, \Delta t}: f_{n} \rightarrow f_{n+1}:=P_{h} \mathcal{T} f_{n}, \quad n=0, \ldots, N-1,
$$

where $h$ denotes the maximal diameter of the elements in $\mathcal{K}_{h}$ and $P_{h}$ is the associated $P_{1}$ interpolation. Besse's analysis consists in decomposing the error

$$
e_{n+1}:=\left\|f((n+1) \Delta t)-f_{n+1}\right\|_{L^{\infty}}
$$

into three parts, namely, $e_{n+1,1}, e_{n+1,2}$ and $e_{n+1,3}$, as follows:

The first part is

$$
e_{n+1,1}:=\|f((n+1) \Delta t)-\mathcal{T} f(n \Delta t)\|_{L^{\infty}},
$$

and is only due to the approximation of the characteristics by the numerical transport operator $\mathcal{T}$. According to the definition of the approximated (13) and exact (12) backward flows, this first error term is bounded by

$$
|f(n \Delta t)|_{W^{1, \infty}}\|\mathcal{B}(n)-\mathcal{B}[f(n \Delta t)]\|_{L^{\infty}} \leq C \Delta t^{3}
$$

as long as the initial data $f_{0}$ are in $W^{1, \infty}\left(\mathbb{R}^{2}\right)$, according to Section 3.1 and the accuracy (16) of the numerical flow. Note that the constant $C$ now depends on the initial data, and on the maximal time $T=N \Delta t$.

The second part is

$$
e_{n+1,2}:=\left\|\left(I-P_{h}\right) \mathcal{T} f(n \Delta t)\right\|_{L^{\infty}},
$$

and corresponds to the interpolation error on $K_{h}$. Using (4), it is estimated by

$$
e_{n+1,2} \leq C h^{2}|\mathcal{T} f(n \Delta t)|_{W^{2, \infty}} .
$$

Since $f_{0}$ is assumed to be in $W^{2, \infty}$, so is $f(n \Delta t)$ according to Section 3.1. Now the question is to know whether the transport operator is stable in this seminorm, which is shown in (Besse, 2004). We therefore have

$$
e_{n+1,2} \leq C h^{2}|f(n \Delta t)|_{W^{2, \infty}} \leq C h^{2} .
$$

Finally, the remainder part is

$$
e_{n+1,3}:=\left\|P_{h}\left(\mathcal{T} f(n \Delta t)-\mathcal{T} f_{n}\right)\right\|_{L^{\infty}},
$$

and is easily bounded by $\left\|\mathcal{T} f(n \Delta t)-\mathcal{T} f_{n}\right\|_{L^{\infty}}$, which can be seen as the propagation of the numerical error at the previous time step. Note that if the operator $\mathcal{T}$ was linear, $\mathcal{T} f(n \Delta t)-\mathcal{T} f_{n}$ would simply read $\mathcal{T}(f(n \Delta t)-$ $f_{n}$ ) and, because a pointwise transport operator can only diminish the amplitude of a function, we would clearly have $\left\|\mathcal{T} f(n \Delta t)-\mathcal{T} f_{n}\right\|_{L^{\infty}} \leq e_{n}$. In fact, we know that the operator $\mathcal{T}$ is nonlinear, but it is stable enough to satisfy

$$
\left\|\mathcal{T} f(n \Delta t)-\mathcal{T} f_{n}\right\|_{L^{\infty}} \leq(1+C \Delta t) e_{n} .
$$

Gathering the above estimates, Besse obtains

$$
e_{n+1} \leq(1+C \Delta t) e_{n}+C\left(\Delta t^{3}+h^{2}\right) \text {, }
$$

which yields, according to the Gronwall lemma,

$$
e_{n} \leq C\left(\Delta t^{2}+h^{2} / \Delta t\right),
$$

where $C$ only depends on the initial data $f_{0}$ and on the final time $T$. Balancing $\Delta t^{3} \sim h^{2}$ in this estimate, we eventually find the following convergence rate:

$$
\left\|f(n \Delta t)-f_{n}\right\|_{L^{\infty}} \leq C h^{4 / 3} \leq C \#\left(\mathcal{K}_{h}\right)^{-2 / 3} .
$$

\section{Adaptive Semi-Lagrangian Scheme}

Following the three steps described in Section 3.2, the principle of the adaptive semi-Lagrangian (AdSL) scheme simply consists in interpolating the transported $\mathcal{T} f_{n}$ on a mesh which is adapted to it rather than fixed.

4.1. Predicting Adaptive Meshes. In order to simplify the "mesh design" as much as possible, we now restrict ourselves to triangulations that can be derived from quad meshes as described in Section 2.3. We shall then associate to any numerical solution $f_{n}$ a quad mesh $M_{n}$. Given the couple $\left(M_{n}, f_{n}\right)$, our problem now consists in building, at each time step $n$, a new mesh $M_{n+1}$ that is well adapted to $\mathcal{T} f_{n}$. In the light of Section 2, one may want to use the $\varepsilon$-adapted mesh $M_{\varepsilon}\left(\mathcal{T} f_{n}\right)$ given by Algorithms 1 and 2. Unfortunately, and as Remark 2 stressed out, $\mathcal{T} f_{n}$ is not to be computed everywhere, so that Algorithm 1 cannot be applied on it. One then has to find another way to guess what the adaptive $M_{n+1}$ should be.

As a starter, here is the prediction strategy that was proposed to us by Eric Sonnendrücker during the CEMRACS 2003 summer school, and implemented in the YODA code described in (Campos Pinto and Mehrenberger, 2005). For an accurate prediction strategy in the context of scalar conservation laws and finite volume schemes, see also (Cohen et al., 2003). Remember that we have at hand a graded quad mesh $M_{n}$ and assume that we know how to compute from the numerical solution $f_{n}$ not only a backward flow $(x, v) \rightarrow \mathcal{B}\left[f_{n}\right](x, v)$, but also the forward one $\mathcal{F}\left[f_{n}\right]:=\mathcal{B}\left[f_{n}\right]^{-1}$. For each cell $\alpha$ in $M_{n}$, we then advect its center $c(\alpha)$ along $\mathcal{F}\left[f_{n}\right]$, and denote by 
$\mathbb{F}\left[f_{n}\right](\alpha)$ the cell of level $\ell(\alpha)$ that contains $\mathcal{F}\left[f_{n}\right](c(\alpha))$ (up to choosing a cell if this point falls on an edge). A reasonable mesh for interpolating $\mathcal{T} f_{n}$ is given by the smallest one containing the set $\left\{\mathbb{F}\left[f_{n}\right](\alpha): \alpha \in M_{n}\right\}$. Clearly, its building cost is about $C_{\mathcal{F}} \#\left(M_{n}\right)$ (where $C_{\mathcal{F}}$ is an upper bound to the computational cost of one position $\mathcal{F}\left[f_{n}\right](x, v)$, see Remark 2), which is of the same order as the computational cost of a numerical solution, provided that $C_{\mathcal{F}}$ and $C_{\mathcal{B}}$ are of the same order. The major drawback is that there is no guarantee that the corresponding interpolation will achieve a given accuracy.

\subsection{Optimal Transport of Multiscale Meshes.}

Based on both the desire of constructing $\varepsilon$-adapted meshes and the will to remain within a building cost on the order of $C_{\mathcal{B}} \#\left(M_{n}\right)$, the algorithm we now describe, and which was first introduced in (Campos Pinto and Mehrenberger, 2007), maps the couple $\left(M_{n}, f_{n}\right)$ to a new graded mesh, denoted by $\mathbb{T}\left[f_{n}\right]\left(M_{n}\right)$, which has the following properties:

- its building cost is of the order of $C_{\mathcal{B}} \#\left(M_{n}\right)$, where $C_{\mathcal{B}}$ is an upper bound to the computational cost of one position $\mathcal{B}\left[f_{n}\right](x, v) \in \Omega$;

- its cardinality is of the same order as $M_{n}$, i.e., $\#\left(\mathbb{T}\left[f_{n}\right]\left(M_{n}\right)\right) \leq C \#\left(M_{n}\right)$, and its maximal level is no greater than the one of $M_{n}$;

- provided that $M_{n}$ is $\varepsilon$-adapted to $f_{n}, \mathbb{T}\left[f_{n}\right](M)$ is $C \varepsilon$-adapted to $\mathcal{T} f_{n}:=f_{n} \circ \mathcal{B}\left[f_{n}\right]$ with an absolute constant $C$.

Remark 5. (optimality of $\mathbb{T}\left[f_{n}\right]$ ) The operator $\mathbb{T}\left[f_{n}\right]$ is said to be optimal because it satisfies

$$
\left\|\left(I-P_{\mathbb{T}\left[f_{n}\right]\left(M_{n}\right)}\right) \mathcal{T} f_{n}\right\|_{L^{\infty}} \leq C \varepsilon \leq C^{\prime} \#\left(\mathbb{T}\left[f_{n}\right]\left(M_{n}\right)\right)^{-1}
$$

provided that $M_{n}$ is $\varepsilon$-adapted to $f_{n}$ and that it satisfies $\varepsilon \leq C \#\left(M_{n}\right)^{-1}$.

Before writing down the algorithm, let us describe it in a few words. Basically, the main idea is to perform recursive splitting like in Algorithm 1, but with a different refinement criterion. As has been mentioned above, the cells that we wish to subdivide are those on which the $W^{2,1}$ seminorm of $\mathcal{T} f_{n}$ is larger than $\varepsilon$, but we do not have this function at hand. Nevertheless, since $\mathcal{T} f_{n}$ reads $f_{n} \circ \mathcal{B}\left[f_{n}\right]$, we may ask whether

$$
\left|\mathcal{T} f_{n}\right|_{W^{2,1}(\alpha)} \leq C\left|f_{n}\right|_{W^{2,1}\left(\mathcal{B}\left[f_{n}\right](\alpha)\right)},
$$

or, in other words, if the numerical transport operator $\mathcal{T}$ is stable with respect to the $W^{2,1}$ seminorm. For the moment, let us assume that the answer to that question is yes (and, as we shall see in Section 4.5, the actual answer is: strictly speaking, no, but essentially, yes). If (18) holds, we can decompose the right-hand side over the cells of $M_{n}$ that intersect $\mathcal{B}\left[f_{n}\right](\alpha)$, i.e.,

$$
\left|\mathcal{T} f_{n}\right|_{W^{2,1}(\alpha)} \leq C \sum_{\beta \in \mathcal{I}\left(M_{n}, f_{n}, \alpha\right)}\left|f_{n}\right|_{W^{2,1}(\beta)},
$$

where the set $\mathcal{I}\left(M_{n}, f_{n}, \alpha\right)$ is precisely defined as

$$
\mathcal{I}\left(M_{n}, f_{n}, \alpha\right):=\left\{\beta \in M_{n}, \beta \cap \mathcal{B}\left[f_{n}\right](\alpha) \neq \emptyset\right\} .
$$

Now, if $M_{n}$ is $\varepsilon$-adapted to $f_{n}$, as it ought to be, every quantity $\left|f_{n}\right|_{W^{2,1}(\beta)}$ appearing in the above sum is precisely bounded by $\varepsilon$. One question then remains, namely: Is it possible to bound the cardinality of the set $\mathcal{I}\left(M_{n}, f_{n}, \alpha\right)$ by a constant? The good news is that this is possible. More precisely, if the center of $\alpha$ is backward advected into a cell of $M_{n}$ whose level is no greater than that of $\alpha$, that is to say, if $\mathcal{B}\left[f_{n}\right](c(\alpha))$ belongs to some $\beta \in M_{n}$ such that $\ell(\beta) \leq \ell(\alpha)$, then we have

$$
\mathcal{I}\left(M_{n}, f_{n}, \alpha\right) \leq C
$$

with an absolute constant, provided that $\Delta t$ is no greater than a constant that depends on $f_{0}$ and $T$. This inequality is deeply connected to the graded property (11) of the mesh $M_{n}$, and to the fact that the numerical flow $\mathcal{B}\left[f_{n}\right]$ is a $C^{1}$-diffeomorphism. Here again, we refer to (Campos Pinto and Mehrenberger, 2007) or, for French readers, to Chapters 5 and 6 in (Campos Pinto, 2005) for a detailed proof. Denoting by

$$
\ell^{*}\left(M_{n}, f_{n}, \alpha\right):=\max \left\{\ell(\beta): \beta \in M_{n}, \mathcal{B}\left[f_{n}\right]\left(c_{\alpha}\right) \in \bar{\beta}\right\}
$$

the level of this cell $\beta$ (or the maximal level over such cells in the case where $\mathcal{B}\left[f_{n}\right]\left(c_{\alpha}\right)$ lies on a edge), what we are able to show is essentially that

$$
\left|\mathcal{T} f_{n}\right|_{W^{2,1}(\alpha)} \leq C \varepsilon \quad \text { as long as } \quad \ell^{*}\left(M_{n}, f_{n}, \alpha\right) \leq \ell(\alpha) \text {, }
$$

up to a few modifications mentioned in Section 4.5 below. In other words, we have just found a computable refinement criterion for designing a mesh that is well adapted to $\mathcal{T} f_{n}$. Our algorithm is then as follows:

Algorithm 3. (optimal mesh transport)

- Starting from $\Lambda_{0}=\mathcal{Q}_{0}=\{\Omega\}$,

- build for $\ell \geq 0$

$$
\begin{aligned}
\Lambda_{\ell+1}:=\Lambda_{\ell} \cup & \{\beta \in \mathcal{C}(\alpha): \\
& \left.\alpha \in M\left(\Lambda_{\ell}\right), \ell^{*}\left(M_{n}, f_{n}, \alpha\right)>\ell(\alpha)\right\}
\end{aligned}
$$

until $\Lambda_{L+1}=\Lambda_{L}$,

- and let $\mathbb{T}\left[f_{n}\right] M_{n}$ be the smallest graded refinement of $\Lambda_{L}$ (see Algorithm 2). 
4.3. Essential Form of the AdSL Scheme. We now have at hand a practical algorithm to build at a reasonable cost a graded mesh $\mathbb{T}\left[f_{n}\right] M_{n}$ that is $C \varepsilon$-adapted to $\mathcal{T} f_{n}$ as long as $M_{n}$ is $\varepsilon$-adapted to $f_{n}$, so that the interpolation error $\left\|\left(I-P_{\mathbb{T}\left[f_{n}\right] M_{n}}\right) \mathcal{T} f_{n}\right\|_{L^{\infty}}$ is of the order of $\varepsilon$. Note that having a multiplicative constant in front of $\varepsilon$ is not a major problem for a few iterations, but that using only Algorithm 3 in the scheme would construct meshes $\mathbb{T}\left[f_{n}\right] M_{n}$ that are only $C^{n} \varepsilon$-adapted to the transported $\mathcal{T} f_{n}$, which is very poor as $\Delta t$ goes to zero. To prevent this accumulation, we need to apply Algorithm 2 as a correction step to guarantee that $M_{n}$ is not only $C \varepsilon$, but it is actually $\varepsilon$-adapted to $f_{n}$.

Essentially, the AdSL scheme is then as follows:

$$
\mathbb{S}_{\varepsilon, \Delta t}: f_{n} \rightarrow f_{n+1}:=P_{M_{n+1}} P_{\tilde{M}_{n+1}} \mathcal{T} f_{n}
$$

for $n=0, \ldots, N-1$. Here $\tilde{M}_{n+1}$ and $M_{n+1}$ are respectively given by Algorithms 3 and 2 (up to the modifications described in Section 4.5 below), following

$$
\begin{aligned}
\tilde{M}_{n+1} & :=\mathbb{T}\left[f_{n}\right] M_{n}, \\
M_{n+1} & :=M_{\varepsilon}\left(P_{\tilde{M}_{n+1}} \mathcal{T} f_{n}\right) .
\end{aligned}
$$

Remark 6. In (Campos Pinto and Mehrenberger, 2007), the form of the scheme is a bit more complicated, which is mainly due to the splitting technique (15) used to approach the characteristic curves. Another complication comes from the use of a truncation operator to guarantee that the numerical solutions-like the exact ones-remain compactly supported in spite of the diffusion induced by the interpolations.

4.4. Error and Complexity Estimates. Here is our main result: up to the modifications mentioned in Remark 6 and in Section 4.5, the adaptive solutions $f_{n}$ computed by the AdSL scheme (20)-(21) satisfy

$$
\left\|f(n \Delta t)-f_{n}\right\|_{L^{\infty}} \leq C\left(\Delta t^{2}+\varepsilon / \Delta t\right),
$$

where the constant $C$ depends only on the initial solution $f_{0}$ and on the final time $T=N \Delta t$ of the simulation, provided that

- the initial solution is in $W^{1, \infty}(\Omega) \cap W^{2,1}(\Omega)$ and has a compact support (see Remark 1);

- the parameter $\Delta t$ is no larger than a constant which only depends on the $L^{\infty}$ norm of $f_{0}$ and on the final time $T$ (this requirement is essential for establishing the bound (19));

- the parameter $\varepsilon$ is no larger than $\Delta t^{2}$ (which might only be a technical requirement, but not very restrictive, since balancing the terms in the above estimate leads to choosing $\varepsilon \sim \Delta t^{3}$ as both parameters tend to 0 ).
We refer the reader to (Campos Pinto and Mehrenberger, 2007) or to Chapter 6 in (Campos Pinto, 2005) for a complete proof of this result.

In addition to this error estimate, we know that the cardinality of the predicted meshes is of the same order as that of the corrected ones, i.e.,

$$
\#\left(\tilde{M}_{n+1}\right) \leq C \#\left(M_{n}\right) .
$$

Now, according to Section 2.2, we know that up to preventing the concentration-of-curvature phenomenon, the complexity of these corrected meshes $M_{n}$ should be monitored by $\varepsilon$ and by the $W^{2,1}$ seminorm (or, better, by the total curvature introduced in Section 4.5 below) of the numerical solutions, but at the present stage we do not know how to rigorously estimate the growth of this quantity as time evolves. Our conjecture is that the cardinality of the corrected meshes $M_{n}$ is bounded by $C \varepsilon^{-1}$. Balancing $\varepsilon \sim \Delta t^{3}$ in the estimate (22) would then yield

$$
\begin{aligned}
\left\|f(n \Delta t)-f_{n}\right\|_{L^{\infty}} & \leq C \varepsilon^{2 / 3} \\
& \leq C\left[\max \left(\#\left(M_{n}\right), \#\left(\tilde{M}_{n}\right)\right)\right]^{-2 / 3},
\end{aligned}
$$

provided that the initial data are in $W^{1, \infty} \cap W^{2,1}$. Note that our "adaptive" estimates require less regularity than the "uniform" one (17) (that only holds provided $f_{0}$ is in $\left.W^{2, \infty}\right)$, which is a classical way to express the superiority of the adaptive methods over the uniform ones.

4.5. A Few Precisions. Now let us mention why the stability (18) does not exactly hold in this way. The first reason is that since $f_{n}$ is piecewise affine, its second derivatives are Dirac measures supported on the edges of a given triangulation, and the $W^{2,1}$ seminorm of $f_{n}$ (as well as that of $\mathcal{T} f_{n}$ ) is not even defined. In a way, this difficulty comes from the fact that, unlike Besse, we do not consider the "dynamic interpolation error" $(I-P) \mathcal{T}$ on the exact $f(n \Delta t)$ but on the numerical $f_{n}$ as it actually happens in the scheme. In other words, instead of bounding the error $e_{n+1}=\left\|f((n+1) \Delta t)-f_{n+1}\right\|_{L^{\infty}}$ by $e_{n+1,1}+A$ with

$A=\|(I-P) \mathcal{T} f(n \Delta t)\|_{L^{\infty}}+\left\|P\left(\mathcal{T} f(n \Delta t)-\mathcal{T} f_{n}\right)\right\|_{L^{\infty}}$

as in Section 3.3, we bound it using

$$
A=\left\|(I-P) \mathcal{T} f_{n}\right\|_{L^{\infty}}+\left\|\mathcal{T} f(n \Delta t)-\mathcal{T} f_{n}\right\|_{L^{\infty}} .
$$

Had we chosen the first approach, there would be no problem about the smoothness of $\mathcal{T} f(n \Delta t)$, but, on the other hand, because Algorithm 1 cannot be applied on the unknown $P_{\tilde{M}_{n+1}} \mathcal{T} f(n \Delta t)$, we would not have been able to perform the correction step (21b) and guarantee that the adaptive mesh $M_{n}$ is always $\varepsilon$-adapted to the solution.

Now this difficulty can be overcome by introducing the total curvature of a function, defined as the total mass 
of its second derivatives seen as Radon measures. By analogy with $B V(\Omega)$, we denote by $B C(\Omega)$ the space consisting of the functions $g$ in $L^{1}(\Omega)$ whose second derivatives are Radon measures of finite total mass, and write

$$
|g|_{B C(\Omega)}:=\left|\partial_{x x}^{2} g\right|(\Omega)+\left|\partial_{x v}^{2} g\right|(\Omega)+\left|\partial_{v v}^{2} g\right|(\Omega)
$$

for the corresponding seminorm. It is then possible to show that the functions in $B C(\Omega)$ are continuous, and that the interpolation error estimate (6) still holds when using the $B C$ seminorm instead of the $W^{2,1}$ one, see Chapter 3 in (Campos Pinto, 2005) or (Campos Pinto, 2007).

The second reason is that when analyzing the seminorm $\left|\mathcal{T} f_{n}\right|_{B C(\alpha)}$, we find out that a second quantity comes into play, namely, the $W^{1, \infty}$ seminorm of $f_{n}$. Now, up to introducing the functional

$$
\nu\left(f_{n}, \alpha\right):=\left|f_{n}\right|_{B C(\bar{\alpha})}+\Delta t \operatorname{vol}(\alpha)\left|f_{n}\right|_{W^{1, \infty}(\alpha)}
$$

and establishing $W^{2, \infty}$ estimates on the electric field involved in the numerical flow $\mathcal{B}\left[f_{n}\right]$, it is possible to prove the following stability result (again, see (Campos Pinto and Mehrenberger, 2007) or (Campos Pinto, 2005) for the proofs):

$$
\nu\left(\mathcal{T} f_{n}, \alpha\right) \leq C \sum_{\beta \in \mathcal{I}\left(M_{n}, f_{n}, \alpha\right)} \nu\left(f_{n}, \beta\right),
$$

so that all estimates stated in the previous sections actually hold, at least when the error indicator $\nu(\cdot, \alpha)$ is used in place of the seminorm $|\cdot|_{W^{2,1}(\alpha)}$.

\section{Acknowledgments}

Once again, I would like to thank Professors Albert Cohen and Eric Sonnendrücker for the competent advise that headed Michel and myself to the present results.

\section{References}

Besse N. (2004): Convergence of a semi-Lagrangian scheme for the one-dimensional Vlasov-Poisson system. SIAM Journal on Numerical Analysis, Vol. 42, No. 1, pp. 350-382.

Besse N., Filbet F., Gutnic M., Paun I. and Sonnendrücker E. (2001): An adaptive numerical method for the Vlasov equation based on a multiresolution analysis, In: Numerical Mathematics and Advanced Applications ENUMATH 2001 (F. Brezzi, A. Buffa, S. Escorsaro and A. Murli, Eds.). Ischia: Springer, pp. 437-446.

Campos Pinto M. (2005): Développement et analyse de schémas adaptatifs pour les équations de transport. Ph.D. thesis (in French), Université Pierre et Marie Curie, Paris.

Campos Pinto M. (2007): $P_{1}$ interpolation in the plane and functions of bounded total curvature. (in preparation).

Campos Pinto M. and Mehrenberger M. (2005): Adaptive numerical resolution of the Vlasov equation, In: Numerical Methods for Hyperbolic and Kinetic Problems (S. Cordier, T. Goudon, M. Gutnic, E. Sonnendrücker, Eds.). Zürich: European Mathematical Society, Vol. 7, pp. 43-58.
Campos Pinto M. and Mehrenberger M. (2007): Convergence of an adaptive semi-Lagrangian scheme for the VlasovPoisson system. (submitted).

Cheng C.Z. and Knorr G. (1976): The integration of the Vlasov equation in configuration space. Journal of Computational Physics, Vol. 22, pp. 330-351.

Cohen A., Kaber S.M., Müller S. and Postel M. (2003): Fully adaptive multiresolution finite volume schemes for conservation laws. Mathematics of Computation, Vol. 72, No. 241, pp. 183-225.

Cooper J. and Klimas A. (1980): Boundary value problems for the Vlasov-Maxwell equation in one dimension. Journal of Mathematical Analysis and Applications, Vol. 75, No. 2, pp. 306-329.

Dahmen, W. (1982): Adaptive approximation by multivariate smooth splines. Journal of Approximation Theory, Vol. 36, No. 2, pp. 119-140.

DeVore, R. (1998): Nonlinear approximation. Acta Numerica, Vol. 7, pp. 51-150.

Glassey R.T. (1996): The Cauchy Problem in Kinetic Theory. Philadelphia, PA: SIAM.

Gutnic M., Haefele M., Paun I. and Sonnendrücker E. (2004): Vlasov simulations on an adaptive phase-space grid. Computer Physics Communications, Vol. 164, No. 1-3, pp. 214-219.

Iordanskii S.V. (1964): The Cauchy problem for the kinetic equation of plasma. American Mathematical Society Translations, Series 2, Vol. 35, pp. 351-363.

Raviart P.-A. (1985): An analysis of particle methods. Lecture Notes in Mathematics, Vol. 1127, Springer, Berlin, pp. 243-324.

Roussel O., Schneider K., Tsigulin, A. and Bockhorn H. (2003): A conservative fully adaptive multiresolution algorithm for parabolic PDEs. Journal of Computational Physics, Vol. 188, No. 2, pp. 493-523.

Sonnendrücker E., Filbet F., Friedman A., Oudet E. and Vay J.L. (2004): Vlasov simulation of beams with a moving grid. Computer Physics Communications, Vol. 164, pp. 390395.

Sonnendrücker E., Roche J., Bertrand P. and Ghizzo A. (1999): The semi-Lagrangian method for the numerical resolution of the Vlasov equation. Journal of Computational Physics, Vol. 149, No. 2, pp. 201-220.

Yserentant H. (1992): Hierarchical bases, In: ICIAM 91: Proceedings of the 2nd International Conference on Industrial and Applied Mathematics (R.E. O’Malley, Ed.). Philadelphia, PA: SIAM, pp. 256-276. 
